\title{
SEISMIC PERFORMANCE EVALUATION OF FLUID VISCOUS
} DAMPERS

\author{
SaiChethan K. ${ }^{1}$, Srinivas K.S. ${ }^{2}$, Ranjitha K.P \\ ${ }^{1} P G$ Student, Department of Civil Engineering, SJB Institute of Technology, Bengaluru, Karnataka, India \\ ${ }^{2}$ Assistant Professor, Department of Civil Engineering, SJB Institute of Technology, Bengaluru, Karnataka, India \\ ${ }^{3}$ Assistant Professor, Department of Civil Engineering, SJB Institute of Technology, Bengaluru, Karnataka, India
}

\begin{abstract}
An earthquake directly affects a structure by increasing the energy within the structural system. A significant portion of this energy can be dissipated by the introduction of structural control systems. Several structural control systems such as passive, active and semi-active control systems are gaining importance nowadays in the earthquake resistant design of structures. Fluid viscous damper (FVD) is a type of passive energy dissipation device, which operates on the principle of fluid flow through orifices. This type of damper has found numerous applications in the military and aerospace industry from many years. Recently, in the civil engineering field, high capacity fluid viscous dampers have found commercial applications on buildings and bridges subjected to seismic and/or wind storm inputs. FVDs use inertial flows, where oil is forced through small orifices at high speeds, in turn generating high damping force. In the present study, the effectiveness of fluid viscous dampers in reducing the responses of a structure under seismic excitations is evaluated analyticallyusing non-linear time history analysis. A twenty story reinforced concrete structure with square plan is considered in this study. Acceleration time histories of Indian seismic zone IV and zone $V$ are used for the analysis. The analysis is carried out using the computer package ETABS 2015. The analysis results confirmed that a significant reduction in the responses such as displacements and other forces is possible with the introduction of fluid viscous dampers and hence it can be used as an alternative to the conventional ductility based design method of earthquake resistant structures.
\end{abstract}

Keywords: Fluid Viscous Damper, Non-linear Time History Analysis, Energy Dissipation

\section{INTRODUCTION}

An earthquake is shaking of earth surface by waves emerging from the source of disturbance in the earth by virtue of release of energy in the earth's crest. The earthquake waves induce a large amount of lateral load on the structures. Due to this, the structures may suffer large deformation or complete collapse depending upon the type of structure, magnitude of earthquake and several other factors. The collapse of structures leads to loss of life and property damage, causing a large amount of financial losses and social sufferings. Hence it is necessary to design the structures to resist the earthquakes. Over many decades, the earthquake resistant design of structures was dependent on material ductility to dissipate the seismic energy induced into the structural systems. The ductility based design may provide life safety, as the structure gives enough warning before absolute collapse, but the damage control cannot be achieved to the required level.Because of the drawbacks of ductility based design, many structural control techniques have been developed over the years and are gaining importance nowadays. By installing some devices, mechanisms, substructures in the structure, the dynamic performance of the structure is adjusted. The structural control systems dissipate the major portion of the seismic energy and reduce the forces on the primary structure, thereby limitingthe structural deformations. Thus, the introduction of structural control systems ensures life safety, as well as damage control to the required level. The structural control systems are classified under three basic headings as passive control systems, active control systems and semi-active control systems. The use of these structural control systems is very much limited in India. In the present study, an attempt has been made to introduce fluid viscous dampers (a type of passive energy dissipation devices) in reinforced concrete buildings situated in Indian seismic zone IV and zone $\mathrm{V}$ for seismic energy dissipation and its effects on the responses of the structure are evaluated analytically.

\section{FLUID VISCOUS DAMPERS}

Among the various energy dissipation devices, fluid viscous dampers have been widely used in the vibration control of various structural and mechanical systems. These dampers were widely being used in the military and aerospace industry for many years and recently been adapted for structural applications in civil engineering. FVDs have the unique ability of simultaneously reducing both deflection and stresses within a structure subjected to a transient. This is because a FVD varies its force only with velocity, which delivers a response that is essentially out of phase with stresses. A modern fluid viscous damper functions at a fluid pressure level of significant magnitude, thus making the damper small, compact and easy to install. This type of damper is generally less expensive to purchase, install and maintain compared to other types of dampers. 


\subsection{Components}

FVD consists of a stainless steel piston rod with a bronze orifice head and a self-contained piston displacement accumulator. The damper cylinder is filled with a compressible viscous fluid (silicone oil) which is generally non-toxic, non-flammable, thermally stable and environmentally safe. A typical longitudinal section of fluid viscous damper is shown in figure-1.

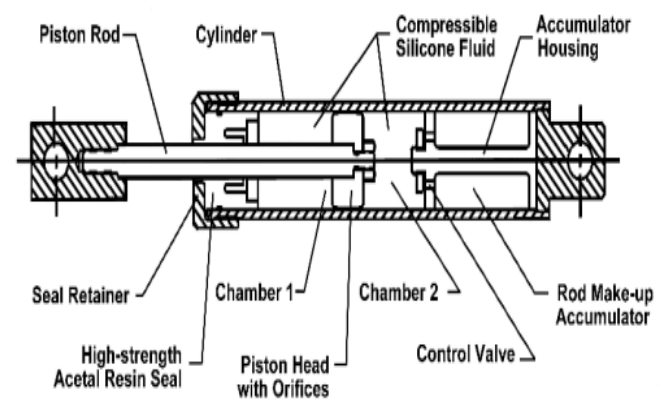

Fig -1: Typical longitudinal section of fluid viscous damper

\subsection{Working}

Fluid viscous dampers work on the principle of fluid flow through orifices. These devices resemble the common shock absorber such as those found in vehicles. FVDs consist of a stainless steel piston that travels through chambers filled with silicone oil. The silicone oil is inert, non-toxic, nonflammable and stable for extremely long periods of time. The silicone oil flows through an orifice in the piston head due to the pressure difference between two chambers and the seismic energy is transformed into heat, which dissipates into the atmosphere. When the FVD is subjected to external excitations, the piston rod with piston will make reciprocating motion in the cylinder to force the damping medium move back and forth between the two chambers separated by the piston. In this process, the friction occurred between the molecules of the damping medium, the medium and the shaft and piston, the damping medium and the cylinder, and the throttling damping force produced by the damping medium through the piston, all these action work together constitute the damping force. The role of FVD is to transform mechanical energy caused by the winds, earthquakes or other structural vibrations into the inner energy of the damping medium. The dampers use the increasing temperature of damping medium to store energy temporarily. The heat is eventually consumed by natural cooling. In this way, the fluid viscous dampers protect the structure from damage.

FVDs can operate over temperature fluctuations ranging from $-40^{\circ} \mathrm{C}$ to $+70^{\circ} \mathrm{C}$.

The ideal damping force of a fluid viscous damper is given by,

$$
\mathrm{F}=\mathrm{C} \cdot \mathrm{V}^{\alpha}
$$

where $\mathrm{F}$ is the damping force, $\mathrm{C}$ is the damping co-efficient, $\mathrm{V}$ is the velocity of piston relative to the cylinder and $\alpha$ is the damping exponent.

\section{DESCRIPTION OF THE ANALYTICAL} MODEL

A 5 bay 20 story reinforced concrete space frame made up of M30 grade concrete and Fe 500 grade steel is considered in this study. A square plan is considered with width of each bay being $7 \mathrm{~m}$. Story height is taken as $3 \mathrm{~m}$. Hence the total width and height of the frame are $35 \mathrm{~m}$ and $60 \mathrm{~m}$ respectively. The support conditions are assumed to be fixed and soil structure interaction effects are neglected. A modal damping of $5 \%$ of critical is considered in all modes in order to account for the material damping. The beam and column sizes are taken as $400 \times 600 \mathrm{~mm}$ and $1000 \times 1000 \mathrm{~mm}$ respectively. Slabs thickness is taken as $150 \mathrm{~mm}$. Wall thickness is assumed as $200 \mathrm{~mm}$, resting on all floor beams and a live load of $4 \mathrm{kN} / \mathrm{m}^{2}$ on all floors and $1.5 \mathrm{kN} / \mathrm{m}^{2}$ on roof are considered. As per IS 1893:2002 (Part 1), a live load reduction factor of 0.5 for all floors except roof is applied in seismic analysis.

As the plan considered is square, the dampers are provided in a similar manner in both directions. Hence the response of the structure will also be the same in both directions. Fluid viscous dampers manufactured by Taylor Devices Inc., USA are considered in this study.Dampers are provided in the peripheral middle bays and in second and fourth bays in the interior frames throughout the height in single diagonal bracings as shown in figure- 2 andfigure-3 respectively. It resulted in a total of 20 fluid viscous dampers in each story and hence a total of 400 dampers in the structure.However, in practice, the available locations for the placement of dampers have to be identified prior to the analysis. Round bar ISRO 100 is considered as extender to mount the fluid viscous damper in the frame.

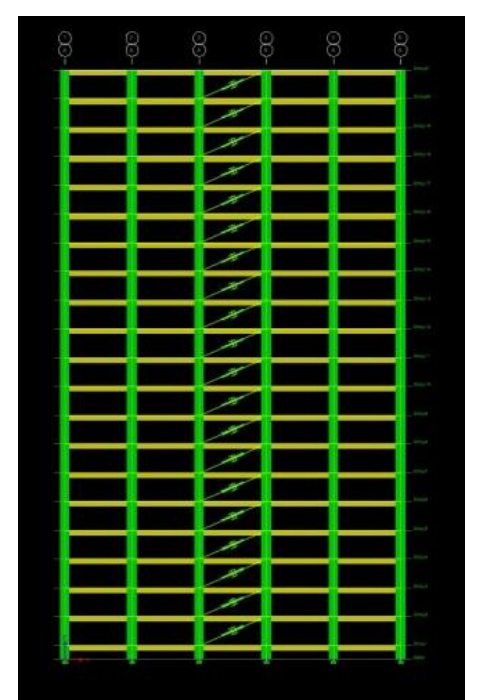

Fig -2: Location of dampers in peripheral frames 


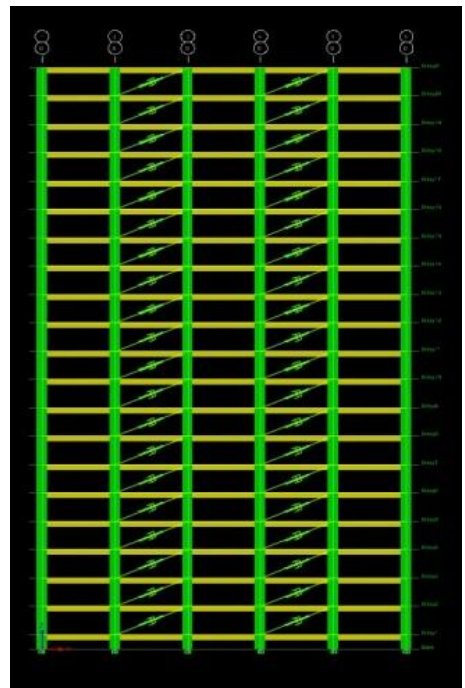

Fig -3: Location of dampers in interior frames

\section{MODELLING AND ANALYSIS}

The modelling and analysis of the bare frame and structure with fluid viscous dampers are done using the ETABS 2015 computer program. The modelling of fluid viscous dampers varies with the type of bracing used to mount the damper to the frame. Link element called Damper - Exponential is used to model fluid viscous dampers. The mass and weight of the damper element along with the bracing connected should be calculated and entered. The fluid viscous dampers mounted in single diagonal bracings are active only in the local axial direction. Therefore, only one active degree of freedom $\mathrm{U} 1$ is selected in the directional properties and since its behaviour is non-linear, the non-linear option is marked. As there will be no provision for rotation, rotational inertia R1, R2 and R3 will be entered zero. After defining the directional properties (U1), the non-linear properties of the damper in that particular direction have to be specified. The values of stiffness, damping co-efficient and damping exponent should be entered in the non-linear properties window. The stiffness will be calculated based on the cross sectional area of the steel section used for bracing and the length of bracing. The value of stiffness is taken as $224400 \mathrm{kN} / \mathrm{m}$ in this study.Non-linear time history analysis is carried out using acceleration time histories of Indian seismic zone IV and zone V.

\section{DETERMINATION OF THE OPTIMAL} PROPERTIES OF DAMPERS

It is important to determine the optimal properties of fluid viscous dampers in order to reduce the response of the structure by a considerable amount. The optimal damper properties are nothing but the values of damping coefficient (C) and damping exponent $(\alpha)$ corresponding to minimum response of the structure and maximum seismic energy dissipation.A simple procedure for the determination of damping co-efficient is used in this study [5].

$$
\mathrm{C}=2 . \mathrm{m} . \omega . \xi
$$

where $\xi$ is the damping ratio, $\mathrm{m}$ is the seismic weight of the structure and $\omega$ is the natural frequency which is given by $\omega$ $=2 \pi / \mathrm{T}$. Here, $\mathrm{T}$ is the fundamental time period, which is obtained from modal analysis. Knowing the value of natural frequency $\omega$, the co-efficient of damping is calculated by assuming suitable value of damping ratio $\xi$ and is used in analysis. The value of damping exponent varies in the range of 0.2 to 1.0 for typical fluid viscous dampers used in structural applications. In the present study, the damping coefficient valuescorresponding to $\xi$ values of $20 \%, 25 \%$ and $30 \%$ are used, and the damping exponent values of 0.2 , $0.3,0.4,0.5$ and 0.6 are used. The seismic weight of the structure $(\mathrm{m})$ and fundamental time period $(\mathrm{T})$ obtained from modal analysis are $324870.4 \mathrm{kN}$ and $2.83 \mathrm{sec}$. respectively.

In this study, maximum displacement of top story, maximum base shear, maximum axial load on the columns and percentage of input energy dissipated by the dampers are considered as the criteria to determine the optimal values of damping coefficient and damping exponent.These response quantities are first determined for bare frame using non-linear time history analysis. The FVDs are then introduced and a series of non-linear time history analysis are carried out by varying the values of damping co-efficient and damping exponent and the above mentioned response quantities are noted. The optimum values of damping coefficient and damping exponent are obtained as $1082 \mathrm{kN}-\mathrm{s} / \mathrm{m}$ and 0.2 respectively for both zone IV and zone $\mathrm{V}$ time histories.It is observed that the percentage of input energy dissipated by the dampers with above mentioned properties in zone IV and zone V are $68.38 \%$ and $66.41 \%$ respectively. In the present study, uniform damper properties are maintained throughout the height of the building. However, it is possible to vary the damper properties along the height in order to optimize the response and also the cost of the dampers.

\section{RESULTS AND DISCUSSIONS}

After obtaining the optimal values of damping coefficient and damping exponent of the fluid viscous dampers, the response quantities are obtained for bare frame and the damped framed and the results are compared. The response quantities considered in this study are maximum story displacements, maximum story drifts, maximum story shears, maximum axial loads in the columns due to earthquake and maximum moments in the columns due to earthquake in each story level. The reduction in each of these responses at each story level is found out and the percentage reduction is also determined for both zone IV and zone V. Since the frame is symmetric in both the directions, the response quantities are also same in both the directions.

The variation of the maximum story displacement, maximum story drift, maximum story shear, maximum axial load in the columns, maximum moments in the columns for bare frame and fluid viscous damped frames under both zone IV and zone $\mathrm{V}$ time histories are shown in Chart -1 to Chart -10 respectively. The average reduction in each of the 
response quantities by the introduction of FVDs in zone IV and zone $\mathrm{V}$ are tabulated in Table -1 .

Table -1: Average reduction in the responses of damped frame

\begin{tabular}{|l|l|l|}
\hline \multirow{2}{*}{$\begin{array}{l}\text { Average reduction } \\
(\%) \text { in }\end{array}$} & Seismic Zone \\
\cline { 2 - 3 } & Zone IV & Zone V \\
\hline Base shear & 38.79 & 33.53 \\
\hline Displacement & 28.15 & 28.37 \\
\hline Acceleration & 30.05 & 31.60 \\
\hline Story drifts & 42.57 & 40.49 \\
\hline Story Shear & 31.76 & 33.50 \\
\hline Column axial loads & 52.47 & 46.59 \\
\hline Column moments & 45.06 & 42.36 \\
\hline
\end{tabular}

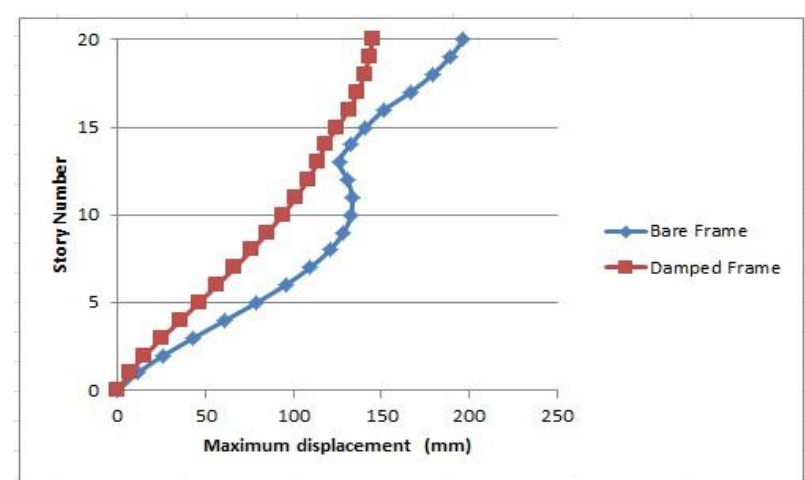

Chart -1: Variation of maximum story displacement in bare frame and damped frame for zone IV

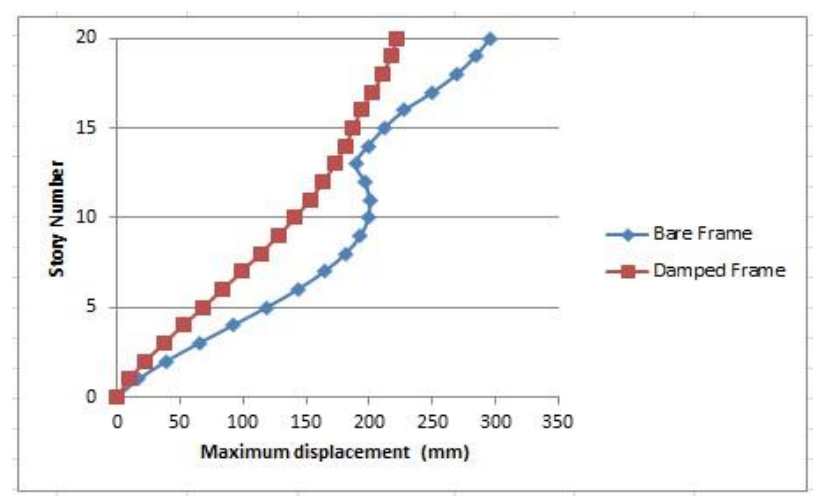

Chart -2: Variation of maximum story displacement in bare frame and damped frame for zone $\mathrm{V}$

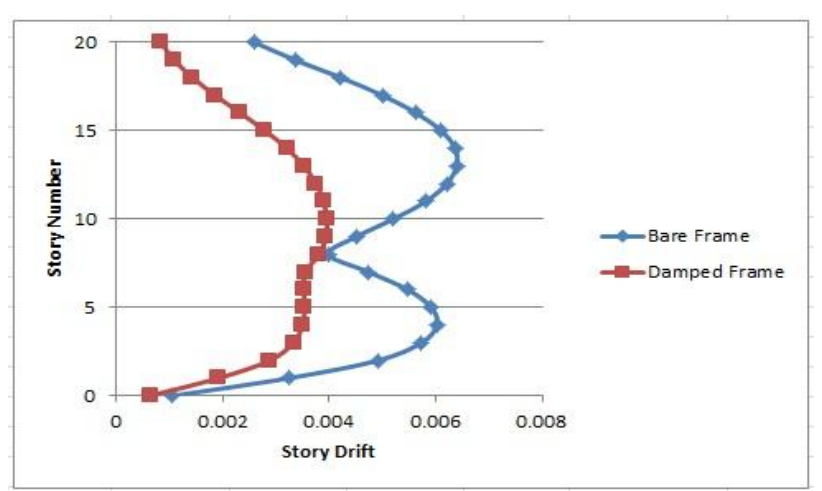

Chart -3: Variation of maximum story drift in bare frame and damped frame for zone IV

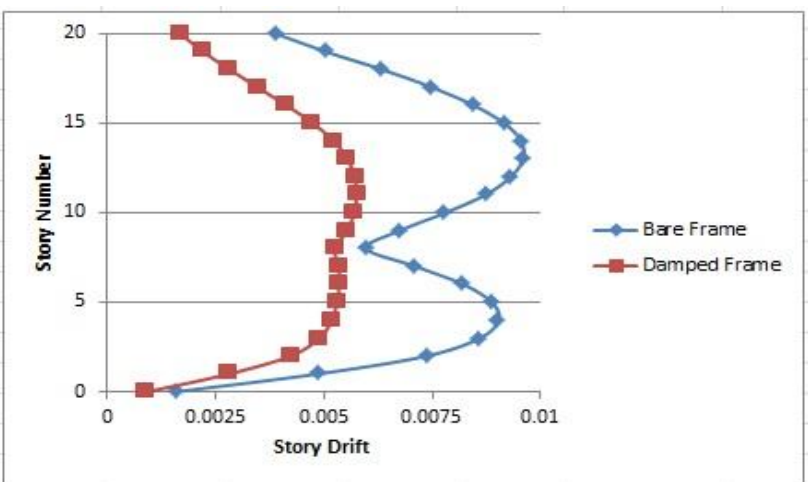

Chart -4: Variation of maximum story drift in bare frame and damped frame for zone $\mathrm{V}$

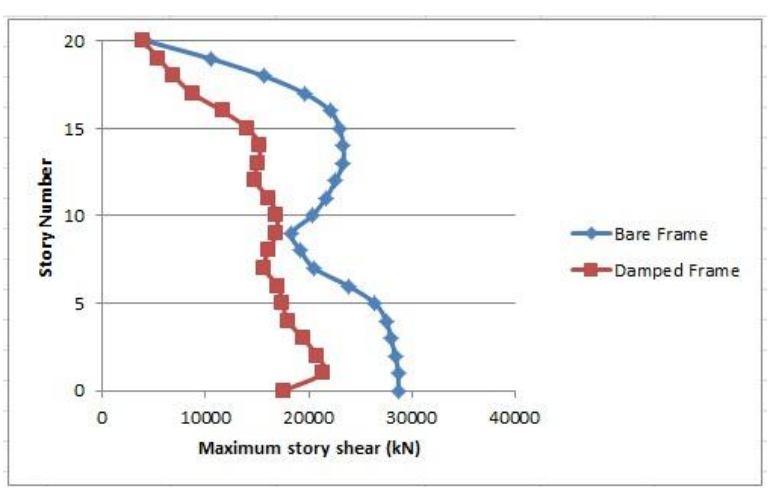

Chart -5: Variation of maximum story shear in bare frame and damped frame for zone IV

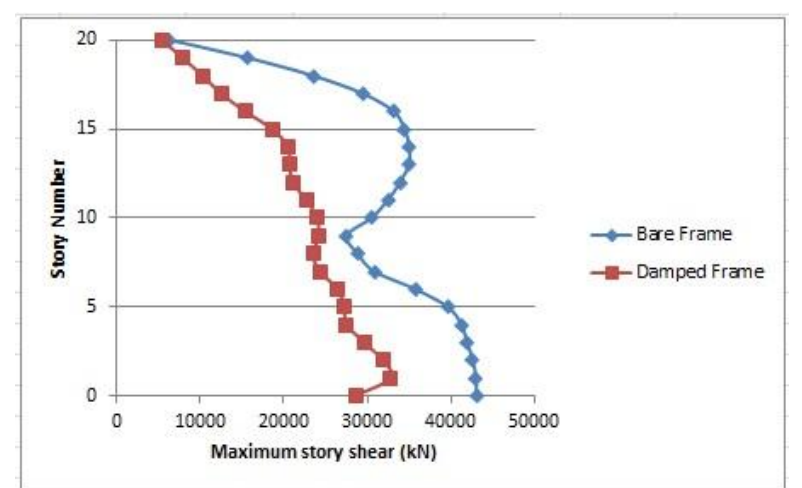

Chart -6: Variation of maximum story shear in bare frame and damped frame for zone $\mathrm{V}$

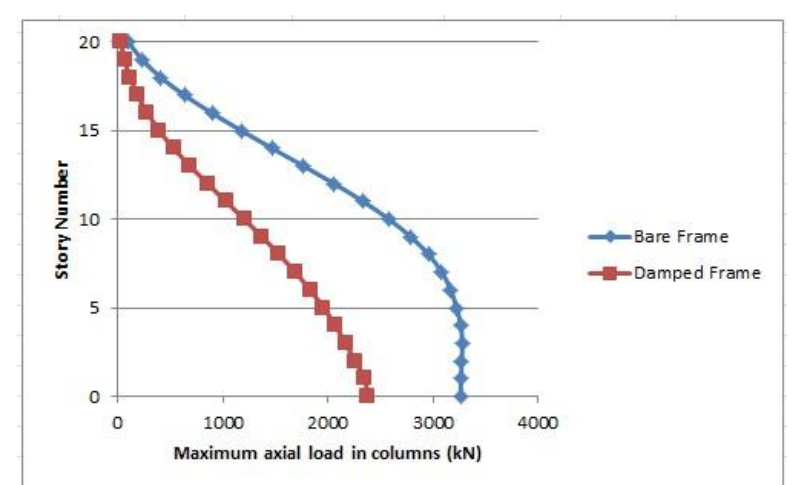

Chart -7: Variation of maximum axial load in the columns in bare frame and damped frame for zone IV 


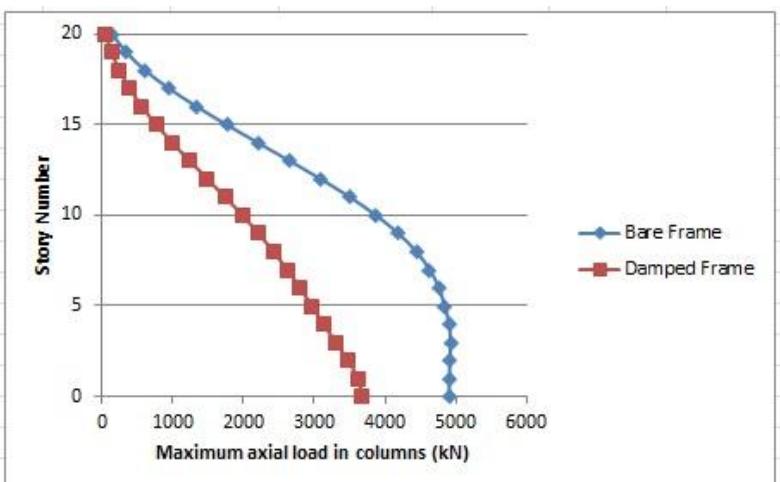

Chart -8: Variation of maximum axial load in the columns in bare frame and damped frame for zone $\mathrm{V}$

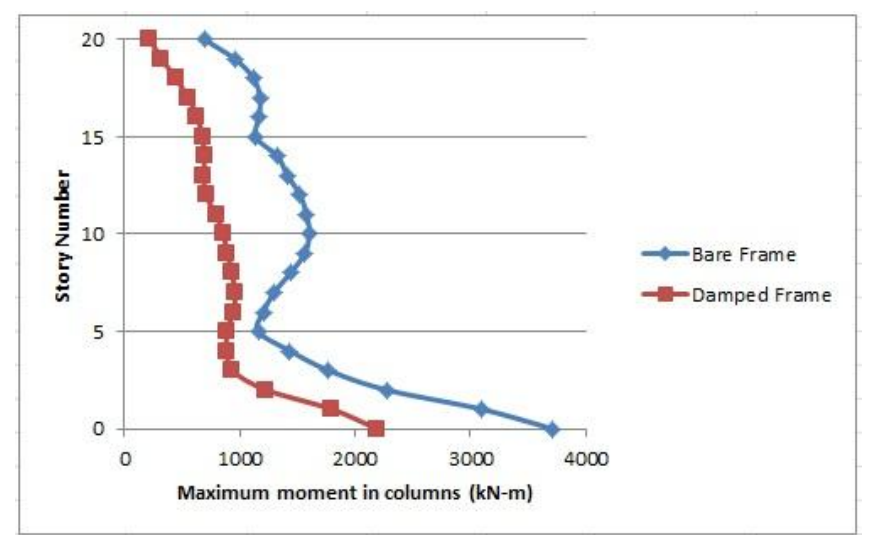

Chart -9: Variation of maximum column moments in bare frame and damped frame for zone IV

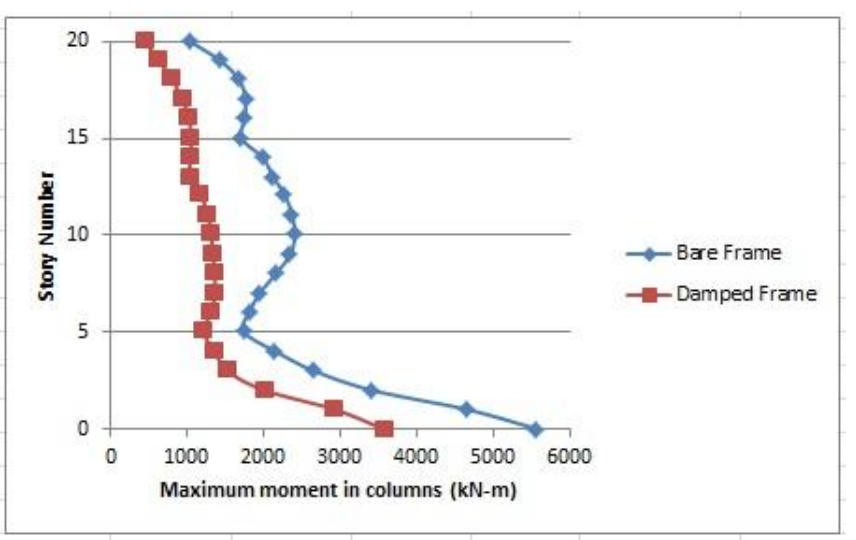

Chart -10: Variation of maximum column moments in bare frame and damped frame for zone $\mathrm{V}$

From the results, it is clear that fluid viscous dampers are efficient in reducing both forces and displacements considerably in both the seismic zones.

\section{CONCLUSION}

From the results of the non-linear time history analysis on the bare frame and damped frame, the following conclusions can be drawn.

- Fluid viscous dampers can dissipate major portion of the seismic energy and hence reduce the energy input on the primary structure.
- The FVDs are capable of reducing both forces and displacements of the structure under seismic loads.

- The response of the structure varies with damping exponent and damping coefficient. By the proper selection of optimum values of these factors, the response of the structure can be minimized.

- It can also be concluded that the fluid viscous dampers can be effectively used as one of the better alternatives for the conventional ductility based design methods of earthquake resistant design of structures.

\section{REFERENCES}

[1] T.T Soong, B.F. Spencer Jr, Supplemental energy dissipation: state-of-the-art and state-of-the-practice, Engineering Structures 24 (2002), 243-259.

[2] Douglas P. Taylor, Fluid dampers for applications of seismic energy dissipation and seismic isolation, Eleventh World Conference on Earthquake Engineering, Paper no. 798.

[3] Douglas Taylor, Philippe Duflot, Fluid viscous dampers used for seismic energy dissipation in structures.

[4] IS 1893 (Part - 1): 2002, Indian Standard criteria for earthquake resistant design of structures - General provisions and buildings, Bureau of Indian Standards, New Delhi.

[5] SP: 6-1 (1964), ISI Handbook for Structural Engineers - Part - 1 - Structural Steel Sections, Bureau of Indian Standards, New Delhi.

[6] Liya Mathew, C. Prabha, Effect of fluid viscous dampers in multi-storeyedbildings,IMPACT: IJRET, Vol.2, Issue 9, Sep 2014, 59-64.

[7] G.S. Balakrishna, Jini Jacob, Seismic analysis of building using two types of passive energy dissipation devices, IOSR Journal of Mechanical and Civil Engineering (IOSR-JMCE), PP 13-19.

[8] YuvrajBisht, SaraswatiSetia, Seismic behavior of a soft storey building with \& without viscous dampers, International Journal of Engineering Research and Applications (IJERA), March 2014.

[9] A. Ras, N. Boumechra, Seismic energy dissipation study of linear fluid viscous dampers in steel structure design, Alexandria Engineering journal (2016) 55, 2821-2832.

[10] A.K. Sinha, Sharad Singh, Structural response control of RCC moment resisting frame using fluid viscous dampers, International Journal of Civil Engineering and Technology (IJCIET), Volume8, Issue 1, Jan 2017, pp. 900-910.

\section{BIOGRAPHIES}

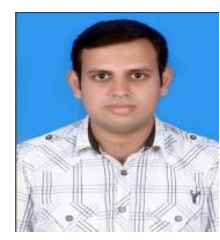

Mr. SaiChethan K, PG Student, Department of Civil Engineering, SJB Institute of Technology, Bengaluru. 


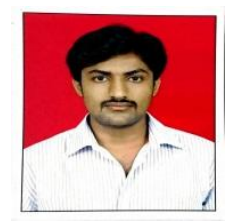

Mr. Srinivas K.S, Assistant Professor,

Department of Civil Engineering, SJB

Institute of Technology, Bengaluru.

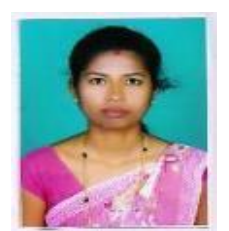

Mrs. Ranjitha K.P, Assistant Professor, Department of Civil Engineering, SJB

Institute of Technology, Bengaluru 